\title{
Un dilemme pratique à trancher depuis des valeurs logées dans l'activité
}

Conséquences pour la connaissance et la professionnalité des éducateurs A practical dilemma to solve through values rooted in activity. Consequences for the knowledge and professionality of special educators

Sylvie Mezzena, Kim Stroumza et Nicolas Kramer

\section{(2) OpenEdition}

1 Journals

Édition électronique

URL : http://journals.openedition.org/activites/2791

DOI : 10.4000/activites.2791

ISSN : 1765-2723

Éditeur

ARPACT - Association Recherches et Pratiques sur les ACTivités

Référence électronique

Sylvie Mezzena, Kim Stroumza et Nicolas Kramer, « Un dilemme pratique à trancher depuis des valeurs logées dans l'activité », Activités [En ligne], 13-2 | 2016, mis en ligne le 15 octobre 2016, consulté le 23 avril 2019. URL : http://journals.openedition.org/activites/2791 ; DOI : 10.4000/ activites. 2791

Ce document a été généré automatiquement le 23 avril 2019.

\section{(c) ()) (9)}

Activités est mis à disposition selon les termes de la licence Creative Commons Attribution - Pas d'Utilisation Commerciale - Pas de Modification 4.0 International. 


\section{Un dilemme pratique à trancher depuis des valeurs logées dans l'activité}

Conséquences pour la connaissance et la professionnalité des éducateurs

A practical dilemma to solve through values rooted in activity. Consequences for the knowledge and professionality of special educators

Sylvie Mezzena, Kim Stroumza et Nicolas Kramer

\section{NOTE DE L'ÉDITEUR}

Article soumis le $1^{\mathrm{er}}$ décembre 2015, accepté le 15 juillet 2016

\section{Introduction}

1 Nous commencerons par présenter les problèmes que pose pour une définition de la professionnalité dans le champ du travail social une conception absolutiste des valeurs dans une perspective d'éthique normative : une dissociation de l'expertise et des valeurs entraîne dans son sillage le risque d'une définition négative de la professionnalité et une difficulté à reconnaître les valeurs en rapport avec la production de connaissances. Nous développerons une conception alternative des valeurs en nous appuyant sur les travaux qui s'inscrivent dans une éthique de l'immanence, et plus spécifiquement en utilisant les travaux du philosophe pragmatiste Dewey. Sa théorie des valeurs comme conduites situées dans des activités, et donc comme pouvant être décrite et faisant partie intégrante de l'expertise, nous permettra de répondre aux problèmes soulevés.

2 L'analyse que nous mènerons dans le champ de l'éducation spécialisée vise ainsi à montrer ce que cette approche peut apporter, comment elle permet de décrire ces valeurs comme parties intégrantes des activités et de l'expertise des professionnels. Cette 
analyse porte sur un moment de colloque d'une équipe éducative dans un foyer avec double mission et décrit les enquêtes pratiques des professionnels durant leurs délibérations. Elle examine comment les valeurs sont logées dans l'activité même, en les considérant empiriquement depuis leur double statut de moyens et de fins ${ }^{1}$. Nous conclurons en abordant quelques conséquences pour la question de la connaissance ainsi que pour la définition de la professionnalité.

\section{Valeurs et professionnalité dans le champ du travail social}

\subsection{Problèmes posés par une éthique normative pour la définition de la professionnalité}

3 Dans la littérature consacrée à la professionnalisation en travail social, nous pouvons constater dans les récents débats une opposition entre valeur et technicité dans les considérations sur ce qu'est l'expertise professionnelle (Aballéa, 2012; Ravon, 2008) : ce qui fonde la professionnalité tend désormais à être problématisé depuis une expertise technique (De Zotti, Dumont, \& Marty, 2007 ; Lansiaux, 2008; Sorel, 2005) et notamment relationnelle (Brichaux, 2001), qui se veut distincte d'une rhétorique historique fondant la professionnalisation dans une revendication des valeurs comme idéaux. Cette séparation entre expertise et valeurs est périlleuse à double titre. D'un côté, concevoir les valeurs comme des idéaux à atteindre pose problème en cas de difficultés ou d'impuissance pratique (ce qui n'est pas extraordinaire en travail social) : non seulement on suggère que les valeurs ne sont pas présentes dans l'action, mais en outre on est enclin à évaluer négativement l'action des professionnels à l'aune de son écart avec cet idéal. De l'autre, considérer l'expertise sans faire une place aux valeurs est particulièrement problématique dans un champ professionnel où l'intervention sociale hérite depuis les politiques sociales de missions consistant justement, notamment, à mettre en œuvre des valeurs sociales. Examinons de plus près les présupposés de cette définition absolutiste des valeurs et les problèmes qu'elle pose pour la professionnalité en travail social.

En travail social, de Jonckheere (2007) constate que les valeurs sont associées à des principes absolus et prédéfinis circulant dans le champ professionnel, vers lesquels l'action devrait idéalement tendre.

«Dans les professions de l'aide, l'éthique (...) est conçue comme une morale proposant des normes d'action. L'éthique est alors ramenée à l'énoncé de principes ou de maximes que le sujet doté, comme chez Kant, d'une volonté bonne met en acte. Elle est censée donner des devoirs permettant de mener une vie méritante et vertueuse en regard de valeurs transcendantes » (de Jonckheere, 2007, p. 106).

Cette éthique normative, précise-t-il, renvoie à un sujet maitrisant ses actes, figure bien discutée par Joas qui a déconstruit ses présupposés (1999) : nous avons affaire à un sujet agissant en dehors de tout contexte, comme si l'environnement n'intervenait pas sur ses actions, et comme s'il agissait en dehors de tout rapport avec autrui en étant maitre de son corps. Partant, c'est aussi le modèle téléologique de l'action qui est discuté: Joas pointe les limites d'une logique linéaire fin-moyen subordonnant le choix des moyens et la réalisation de l'action à des fins décidées au préalable et statiques, comme si leur mise en œuvre était indépendante de toute circonstance et n'était pas affectée en cours de route par ce qui se passe dans l'activité. En renvoyant l'action à des idéaux prédéfinis en 
dehors et en amont de son déroulement (comme si les valeurs étaient extérieures à l'action), cette conception absolutiste des valeurs produit des effets négatifs en termes de définition de la professionnalité : soit en confrontant les professionnels à leurs limites voire impuissance en cas de difficulté ou d'échec, «ce qui ne peut que conduire les humains se définissant en tant que sujets moraux à développer des sentiments de culpabilité et d'impuissance» (de Jonckheere, 2007, p. 106); soit en prodiguant des critères d'évaluation de la pratique qu'elle ne pourra que rarement satisfaire parce qu'étant définis en dehors de ses conditions d'effectuation (Mezzena, 2012).

5 De Jonckheere (2007) constate également que les valeurs renvoient à l'intériorité personnelle des professionnels sous forme de normes intériorisées ${ }^{2}$. En considérant que les valeurs n'interviennent dans l'action que sous une forme intériorisée, privée, d'idéaux abstraits, cette éthique normative pose un deuxième problème pour la définition de la professionnalité dans ce champ. Cette approche est en effet problématique pour une profession où la relationnalité fait souvent l'objet d'interprétations qualifiant sa nature d'intuitive, en l'associant à du "feeling» ou à un sixième sens. En réduisant ce phénomène à une chose intérieure non seulement inobservable, mais également indicible, ces interprétations présupposent quelque chose de déjà-là en soi, que l'on aurait ou pas. En tendant du coup la perche à l'innéisme, elles desservent la reconnaissance d'une expertise professionnelle en ne permettant pas de rendre compte des connaissances construites à l'occasion d'apprentissages, tout en en faisant un objet problématique pour la formation.

6 Cette définition internaliste pose cette fois le problème des valeurs sous l'angle de leur description. Elle fait écho à la distinction traditionnelle entre faits et valeurs dans la rhétorique scientifique des sciences sociales: opposition entre des normes définies comme règles, principes ou lois universalisables et extensibles à l'usage public et pouvant être objet de discussion rationnelle; et les valeurs comme relevant du «désirable, du préférentiel et du particulier », et ne pouvant faire l'objet de discussion rationnelle de par leur caractère non universel (Bidet, Quéré, \& Truc, 2011). Cette conception classique renvoie encore au dualisme entre objectivité et subjectivité, ou à la séparation entre pensée et action. Dans ce débat, les faits considérés comme observables peuvent faire l'objet d'une description objective donnant lieu à une production de connaissances, tandis que les valeurs, associées à des états intérieurs et privés, ne peuvent être objectivement étudiées.

7 Au final, la définition des valeurs en opposition à l'expertise engendre deux problèmes : celui d'un risque d'évaluation négative de la professionnalité en cas de difficultés pratiques et celui de l'impossibilité de constituer les valeurs comme source et objet de connaissance. Dans les deux cas, définies de la sorte de façon normative en étant considérées ni comme observables ni comme descriptibles (et donc comme non transmissibles), les valeurs ne trouvent pas de place dans la définition de l'expertise professionnelle. Or, les valeurs sont omniprésentes dans les discours des professionnels, qu'ils s'adressent aux usagers, à leurs pairs, aux politiques ou encore aux partenaires institutionnels. De plus, ce champ professionnel fait l'objet de très nombreuses sources de prescriptions, notamment depuis des codes déontologiques, ce qui renforce l'inscription de sa mise en œuvre dans une éthique prescriptive. Définir l'expertise dans une éthique normative, qui en vient (paradoxalement) à se définir sans faire de place aux valeurs, isole cette expertise de son inscription dans les débats historiques du champ, mais également des discours et préoccupations actuels des professionnels. 
8 Pour toutes ces raisons, nous avons cherché une alternative à la définition absolutiste et normative des valeurs comme idéaux, afin de les intégrer pleinement à notre modélisation de l'activité professionnelle, et partant à la définition de la professionnalité en travail social. Pour pouvoir décrire finement la manière dont les valeurs interviennent dans les activités des professionnels, nous nous sommes tournés vers des travaux qui développent une autre éthique, et plus spécifiquement la philosophie du pragmatisme américain de Dewey, dont la particularité est de ne pas séparer de façon dualiste pensée et action, ou encore connaissance et valeur ${ }^{3}$. En s'inscrivant dans une éthique de l'immanence, la conception deweyenne des valeurs comme conduites nous paraît en effet féconde pour répondre aux problèmes posés par une éthique normative à la définition de la professionnalité dans le champ du travail social.

\subsection{Une alternative avec l'éthique de l'immanence : des valeurs comme conduites situées et observables}

9 L'éthique de l'immanence est une alternative à l'approche absolutiste des valeurs (Bidet, Quéré, \& Truc, 2011 ; Dewey, 2011 ; Garreta, 2007 ; de Jonckheere, 2007), car elle permet de les concevoir autrement en les resituant dans le monde et l'expérience. Les valeurs définies ici comme étant ce qui nous importe, ce à quoi l'on tient, trouvent leur source dans l'expérimentation d'événements et de nos tentatives pour transformer leurs conséquences. Une telle éthique nous intéresse du point de vue de la construction de la professionnalité, car elle permet de problématiser les valeurs en resituant leur formation dans l'action, et de « décrire les problèmes. (...) Dès lors, l'éthique prend la forme d'une pratique de mise en problème » (de Jonckheere, 2007). Garreta prend lui aussi cette voie pragmatiste et à partir de ses travaux sur Dewey, il qualifie de «située » une telle éthique :

«Pour Dewey, la morale, ou plutôt les divers processus de "moralisation" (au sens descriptif, non-moral du terme), sont intimement liés aux conditions et aux conséquences de la conduite, et à l'incorporation dans les conduites subséquentes des "enseignements" tirés des situations précédentes » (2007, p. 27).

Dans la théorie de la valuation deweyenne, les valeurs sont conçues comme des activités concrètes qui sont intrinsèques à des processus d'enquête pratique. Dans la théorie de l'enquête (1938/1993), Dewey définit l'enquête pratique comme toute activité d'un organisme consistant à transformer l'environnement depuis ses rapports mutuels avec lui, afin de parvenir à résoudre les problèmes que la recherche de satisfaction de ses besoins ne manque pas de générer (besoins qui dans le champ du travail social sont liés à la mission d'agir envers autrui) (Mezzena, Stroumza, Seferdjeli, \& Baumgartner, 2013). Enquêter consiste à expérimenter concrètement des rapports avec l'environnement et à en apprécier les effets, tout en évitant certains risques. L'activité se constitue en partenariat avec l'environnement - premier postulat de Dewey- depuis un rapport moyens-fins (plutôt que fins-moyens) : les moyens pour agir se trouvent en situation, dans des ressources que l'environnement nous met à disposition et qu'il s'agit de tester en les utilisant, en s'agençant avec elles depuis nos rapports mutuels ${ }^{4}$. On « fait avec » ces ressources pour parvenir progressivement à nos fins. Les moyens ne sont pas prédéfinissables mais se rencontrent, se découvrent sur le moment et s'expérimentent en agissant. Ils n'existent pas d'emblée comme tout prêts et en attente d'être utilisés, mais se constituent au fil des transformations opérées par l'activité. Dans cette dynamique 
pratique, c'est depuis ce rapport aux moyens que des fins (intermédiaires) sont créées à partir des effets produits au fur et à mesure par l'activité.

Une visée stable guide cette recherche continue d'effets tout en permettant de s'ajuster sur le moment aux circonstances des différentes situations. Si c'est une dynamique de rapport moyens-fins qui prime, en rendant possibles des réponses situées, ajustées à ce qui se passe, en même temps cette (ré)organisation continue des ressources de l'activité est polarisée par une recherche d'effets stables qui garantit l'orientation de l'activité dans le sens de la mission. Ce travail de perspective peut se définir comme un cap à tenir dans la continuité de l'expérience - second postulat de Dewey: les effets recherchés sont toujours les mêmes, même s'ils sont mis en œuvre différemment selon les situations. Il y a ainsi une perspective qui peut progressivement s'instaurer malgré les variations à l'œuvre dans l'activité. Selon les enchaînements d'agencements, une direction spécifique est prise, qui permet de (re) mettre l'activité dans la ligne de la perspective poursuivie.

L'enquête pratique consiste ainsi à investiguer très concrètement, dans le temps même du déroulement de l'activité, des rapports avec les autres «choses» de l'environnement pour d'une part cerner à quel problème on a affaire, et d'autre part orienter la transformation de ce dernier pour qu'il évolue dans le sens de sa résolution. L'activité est ainsi pleine de valeurs comme conduites: pas seulement comme contenus de qualités attribuées, mais aussi comme manières de construire des problèmes en les orientant dans une certaine direction, depuis la formation de «ends-in-view» ou fins intermédiaires comme directions à déterminer en cours d'action. La valeur se définit alors « comme guide pour le jugement et l'action » (Ogien, 2013, p. 8), avec cette idée chère à Dewey que " "ce qui fait valeur" se dévoile à mesure que les individus découvrent ensemble "ce à quoi ils tiennent" dans une situation d'action précise ». Dewey substitue ainsi à l'approche absolutiste des valeurs une définition pratique logeant leur constitution dans l'activité même, dans les situations: «les valeurs se constituent dans le contexte de l'activité dans laquelle l'usage qui en est fait se réalise » (ibid.).

Dewey conçoit ainsi les valeurs comme des conduites sociales donnant lieu à des interactions dans l'espace public, produisant des effets manifestes chez autrui, et ce faisant, pouvant être observables, discutables et révisables. Il les définit comme n'étant "pas une chose d'un type particulier, mais une qualité attribuée, dans certaines conditions et avec certaines conséquences, à un événement, une situation, un objet ou une personne qui existe indépendamment $d u$ fait de recevoir une valeur. Cette attribution n'est pas un acte mental et la valeur ne procède pas d'un processus interne " (Bidet, Quéré, \& Truc, 2011, p. 29). Autrement dit, les valeurs se forment dans des situations et leur formation dépend des actions qui s'y déroulent. En logeant les valeurs non pas dans les personnes, mais dans les activités, et plus précisément dans les rapports mutuels avec l'environnement, «c'est bien pour souligner que les choses n'acquièrent une valeur que dans leurs relations, leurs connexions et leurs transactions de toutes sortes, avec d'autres choses, et non dans leur relation avec un sujet ou une conscience » (Bidet, Quéré, \& Truc, 2011, p. 30). Dewey reconnait donc comme propriété aux valeurs le fait d'être situées, de se produire "dans le monde, dans les "faits spatio-temporels observables" que sont nos activités quotidiennes " (ibid). En définissant les valeurs comme des faits publics en tant que conduites sociales se formant et s'actualisant manifestement dans l'action, Dewey fait coup double: non seulement il propose une alternative à la définition aprioriste des valeurs comme prescriptions idéales transcendant l'expérience et ne permettant pas de décrire les pratiques, mais de plus il 
évite l'écueil d'une définition internaliste des valeurs qui les réduirait à un phénomène privé inaccessible à l'observation.

Bidet, Quéré et Truc (2011) soulignent la « portée éthique des conceptions de Dewey sur la valeur : ce à quoi nous attribuons de la valeur n'est rien d'autre que ce à quoi nous tenons, c'est-à-dire à quoi ou à qui nous manifestons concrètement notre attachement, à travers nos attitudes actives, nos comportements, nos façons d'agir. Nous y tenons: nous le désirons, nous nous efforçons d'y parvenir, de l'obtenir et, lorsque nous l'avons, nous faisons tout pour le conserver et en prolonger l'existence. Car ce à quoi nous tenons est aussi ce par quoi nous tenons " (pp. 33-34). Les valeurs sont à la fois ce à quoi et ce par quoi les professionnels tiennent dans la perspective de la mission à poursuivre. Cette définition pragmatiste des valeurs nous intéresse du point de vue des apports et des conséquences pour la professionnalité. La formation des valeurs depuis l'activité est une voie alternative pour penser l'engagement des professionnels non pas (seulement et d'abord) de manière idéologique, mais pratique, expérientielle, en les localisant dans les accomplissements mêmes.

Dans le processus d'enquête, les valeurs ont un double statut logique de moyens et de fins (Dewey, 2011 ; Bidet, Quéré, \& Truc, 2011). Du point de vue des agencements, synonymes de situations ${ }^{5}$, ce sont des qualités attribuées aux objets et aux relations que l'on entretient avec eux depuis des appréciations situées, immédiates. Ces valeurs attribuées sont des moyens pour construire sur le moment les problèmes pratiques en testant des situations et en appréciant les conséquences pour la mission, en sentant depuis la totalité de l'expérience si la direction prise par l'activité va dans le sens des effets recherchés (Mezzena, Stroumza, \& Kramer, 2016). Du point de vue de la perspective, ce sont également des directions privilégiées, depuis des séries d'agencements ou des rapports moyens-fins que l'on cherche à favoriser et qui alimentent des régularités : il s'agit des valeurs comme ce à quoi tiennent les professionnels, des manières de "priser", qui opèrent sous forme de fins visées comme des guides dans le travail de perspective. Les valeurs, comme habitudes de partenariat avec l'environnement ${ }^{6}$, en concrétisant ce à quoi tiennent les professionnels, expriment la durée, la régularité dans les enquêtes et elles se sont stabilisées parce qu'elles ont fait leur preuve. Les valeurs se forment ainsi à la fois en appui sur des expériences antérieures, dans la continuité de l'expérience de l'action, et dans des appréciations situées à partir des conditions actuelles des problèmes.

\section{Un dilemme pratique à trancher en colloque}

\subsection{L'enquête pratique des éducateurs à la loupe}

16 Durant le moment de colloque analysé, l'équipe doit travailler à la mise en place d'une nouvelle mission, qui consiste à créer des ateliers d'insertion à côté de la première mission initiale d'hébergement et d'accompagnement éducatif au quotidien. À cette occasion un maitre socio-professionnel (MSP) a été engagé pour créer un atelier d'activité de production, à côté d'ateliers de développement personnel pris en charge par les éducateurs du foyer. Les délibérations portent sur la durée limite de fréquentation des ateliers et de ses conditions de renouvellement. Le MSP n'a pas encore démarré son atelier, tandis que les éducateurs viennent d'expérimenter un trimestre d'ateliers de développement avec quelques jeunes résidant au foyer. Il s'agit donc pour l'équipe, en compagnie du responsable d'équipe et d'un membre de la direction, de faire le bilan de 
ces trois premiers mois d'expérience préalable à l'arrivée du MSP, afin de stabiliser un règlement pour le dispositif d'insertion. Cette délibération prend place dans quatre journées consécutives de colloque intensif qui visent à finaliser la nouvelle mission et à la formaliser dans le concept pédagogique. Dans cette activité, il s'agit pour les professionnels de trancher un dilemme. Les professionnels se trouvent pris dans une activité comprenant des tensions qui la tirent dans différentes directions depuis une multitude d'enjeux; directions qui ne vont pas forcément ou d'emblée dans le sens de leur mission.

17 Les éducateurs repartent du projet initial élaboré par un groupe de travail en amont du changement de mission. Le membre de la direction rappelle que «quand l'équipe de projet a pensé au centre de jour, on avait prévu trois mois renouvelables une fois ». Il s'agit pour les éducateurs d'apprécier les conséquences de cette proposition et de la reprendre depuis la réalité de leur pratique, donnant lieu à une perspective propre. Cette idée de "trois mois renouvelables" ne peut pas simplement ou directement être appliquée en étant insérée telle quelle dans l'activité, mais doit être investiguée, appréciée depuis ses effets pour être transformée afin de pouvoir être concrètement intégrée à leur expérience. L'examen détaillé des enquêtes menées en colloque pour mettre en place la nouvelle mission montre que les éducateurs apprécient au fur et à mesure ce qui se passe dans le cours de l'activité depuis une certaine perspective qui les amène à rechercher activement certains effets : il s'agit de transposer de leurs épaules à celles des jeunes un mouvement de prise en charge qui soit porté par le jeune lui-même et dans la durée. Ce n'est pas par choix théorique ou par hasard que les éducateurs évoquent un travail de maturation (terme utilisé par l'équipe), plutôt que d'autonomisation ou de développement. La mission invite les éducateurs à tenter, tant que faire se peut, de reprendre le travail éducatif parental qui a été interrompu, en essayant de le mener le plus loin possible, dans le temps assez court de deux voire trois ans qui leur est imparti avant la fin du placement. La notion de maturation, un terme de nature biologique, exprime cette idée que les éducateurs prennent le relais du milieu familial pour accompagner le développement de croissance normale, en produisant les meilleures conditions possibles pour que ce développement puisse se poursuivre. La mise en mouvement initiée au foyer doit alors être portée par le jeune lui-même, et idéalement à terme être fondée sur des habitudes pour pouvoir perdurer dans le temps.

18 La poursuite de leur perspective les amène à enquêter pour construire, transformer le problème du renouvellement de la fréquentation des ateliers d'une certaine manière qui favorise les effets recherchés et lui permette de s'intégrer à cette perspective de la maturation. Il s'agit de trouver une manière de garantir certaines conditions pour favoriser l'atteinte des effets recherchés, et cela demande aux éducateurs un effort qui se concrétise par le travail d'enquête. La perspective n'est pas définie telle quelle dans les textes de prescription, ni présentée formellement par les éducateurs. La perspective comme visée pratique est expérientielle : c'est à force de faire l'expérience de leur activité que les éducateurs ont appris quels effets ils ont intérêt à rechercher pour mettre en œuvre leur mission. Les effets du travail de perspective sont découverts dans l'après-coup de l'action par les professionnels, depuis les conséquences de ce qui a été fait. Ainsi la perspective ne se joue pas sur des situations isolées, mais est sans cesse travaillée depuis le cumul de situations traversées et prises globalement.

Dans le cours des échanges, les expérimentations, les appréciations et les anticipations de risques ${ }^{7}$ s'entremêlent au fil de l'avancement de l'enquête. Au fur et à mesure de leurs 
délibérations, les professionnels soupèsent des alternatives pour la suite, en expérimentant en idées des voies d'action et en appréciant les effets potentiels de telle ou telle possibilité, en anticipant également certains risques pour empêcher au maximum que l'activité ne s'oriente dans des directions non désirées. La description des conséquences potentielles liées au fait de mettre ou non des limites temporelles au renouvellement des ateliers trouve ainsi sa signification dans l'expérience des éducateurs, qui se confrontent à des situations éducatives comprenant une inertie importante et les confrontant à de l'impuissance.

Un éducateur apprécie une autre conséquence, « on perd notre crédit aux yeux des autres aussi ", en référence aux partenaires institutionnels qui décident des placements au service de protection de la jeunesse. Il s'agit d'éviter le risque de faire durer des placements qui restent sans effet, qui perdent tout sens éducatif en étant maintenus et qui desservent l'image de l'équipe sur l'extérieur. Le problème du renouvellement de la fréquentation des ateliers est ainsi construit en étant défini en appui sur l'expérience antérieure des éducateurs, qui anticipent des risques majeurs connus pour la mission qu'il s'agit d'éviter à l'avenir. Si le renouvellement doit être formalisé dans le concept pédagogique, en même temps il n'existe pas d'emblée une solution a priori toute prête que l'on appliquerait à ce problème. Une remarque d'un éducateur résume alors bien la nature de cette activité consistant à déterminer combien de temps un jeune devrait fréquenter les ateliers : "C'est difficile de le présager », et toute l'activité de délibération va consister à essayer malgré tout de cerner et d'évaluer ce problème en expérimentant des voies pour l'amener à sa résolution.

21 Par la suite, plusieurs autres risques sont encore anticipés depuis l'appréciation d'autres voies. Premièrement, il s'agit d'éviter à tout prix de revenir à une situation bien connue des éducateurs dans ce foyer avant la mise en place de la nouvelle mission, à savoir se retrouver avec des jeunes dont l'insertion aux ateliers ne s'est pas avérée efficace pour les faire bouger, et qui se retrouvent en situation d'oisiveté au foyer (ce qui ramène le problème de ces jeunes à un état originel que l'on cherche justement à transformer, c'està-dire une situation de rupture de formation qui se pérennise).

«Si c'est ceux de l'interne et puis que ça joue pas à l'atelier au bout de trois mois, mais qu'ils ont un hébergement ici, on les a ici alors sur les bras sans rien (...) et puis alors ça veut dire qu'on l'a ici et puis plus en atelier, mais on l'a ici sans rien, ça va pas ».

Deuxièmement, le dilemme se complexifie encore depuis l'anticipation d'un autre risque en rapport avec le partenariat institutionnel et la question des admissions : il s'agit pour les éducateurs de s'appuyer sur un règlement pour négocier avec les partenaires institutionnels et faire opposition à des demandes de placement jugées risquées selon l'état du groupe de jeunes. Et cet aspect s'avère d'autant plus décisif qu'à l'avenir le centre de jour serait susceptible d'accueillir des jeunes externes ne résidant pas au foyer afin de répondre au besoin accru de prestation d'insertion pour jeunes en situation de rupture de formation.

Du coup, les éducateurs trouvent une utilité à la formalisation d'un règlement pour le dispositif d'insertion, car il faut éviter de ne pas mettre de limite temporelle au renouvellement de la fréquentation des ateliers. Dans ce cas, il ne serait plus possible de stopper une insertion qui s'avérerait inefficace, d'exclure un jeune perturbant le déroulement des ateliers et mettant à mal l'insertion des autres jeunes. Et dans la foulée, il s'agit d'éviter que la mission éducative soit menée indépendamment de celle des ateliers, et qui ferait du foyer le lieu où recueillir les jeunes pour lesquels la prestation 
d'insertion n'a pas porté ses fruits. Le membre de la direction rappelle la prescription au sujet des deux missions du foyer et des ateliers : "C'est une seule identité ", ce qui signifie concrètement, comme le dit une éducatrice, que «c'est un pack (...) s'il \{le jeune\} ne va pas à l'atelier il ne va pas à l'hébergement non plus ", à quoi le responsable d'équipe conclut encore que "ça remet en cause sa place effectivement à l'hébergement ». Se trouve alors en jeu dans cette délibération l'intégration de la mission d'insertion avec la mission initiale. Et les professionnels de décrire cette limite communément reconnue dans l'équipe, comme le montrent les enchaînements suivants : "Je prends juste $Y$ \{un ancien résident\} ou $Z$, mais il $y$ en a eu d'autres, parce qu'ils sont mineurs parce que ci parce que ça (...) on veut pas le mettre dehors ", "c'est ce qu'on vit depuis toujours ", " non non non, mais ça, il faut que ça s'arrête ", " on doit plus vivre ça avec les ateliers sinon y a pas de sens, sinon ça a pas de sens ", "c'est pour ça que faut marquer \{dans le concept pédagogique\} ", et le membre de la direction de conclure : «justement, c'est bien pour ça ».

Dans le même temps, quand le MSP propose de fonctionner par contrat, est souligné par le membre de la direction le risque de trop formaliser :

«Il faut faire attention avec les contrats parce que les contrats sont des outils qui peuvent aussi terriblement nous piéger, faut voir (...) à qui ça sert le contrat ».

Il s'agit ainsi pour les éducateurs de ne pas « se coincer » comme ils disent en mettant des règles de renouvellement trop rigides, car selon l'évolution du jeune, prolonger la fréquentation des ateliers leur permet de suffisamment gagner en maturité pour pouvoir enfin se confronter à des situations réelles. Comme le fait remarquer le membre de la direction, « on voit aussi qu'on part de très bas avec $\mathrm{X}$ \{un jeune avec qui les éducateurs ont rencontré de grandes difficultés jusqu'à récemment\}, et puis qu'au bout de trois mois il y a des petits changements qui s'amorcent, mais qu'il est pas prêt à partir à l'extérieur ». Il s'agit donc d'éviter d'être trop rigide en instaurant une règle limitant le renouvellement, et ce faisant ne permettant pas de redéfinir l'action éducative en gardant une marge de manœuvre avec une souplesse temporelle afin de s'ajuster au besoin de temps supplémentaire des jeunes pour gagner en maturation. L'enjeu cette fois-ci consiste à être suffisamment ouvert dans la définition des problèmes pour laisser une chance aux jeunes.

Ces risques renvoient à des enjeux qui tirent l'activité dans des directions qui ne vont pas toujours dans le même sens, et pour lesquels il n'y a pas d'emblée une bonne réponse à apporter, et de surcroît une fois pour toutes, à appliquer depuis des valeurs, idées ou théories prédéfinies. Résoudre ce dilemme consiste très concrètement à trouver une manière pour tout à la fois, en faisant tenir ensemble ces directions: ne pas faire perdurer des placements avec des jeunes qui « restent sur les bras » des éducateurs, dont l'action éducative reste sans effet; mais en même temps maintenir une flexibilité temporelle pour que le jeune puisse prendre le temps qu'il lui faut pour maturer; et le tout en sauvegardant une marge de manœuvre dans les négociations avec les partenaires institutionnels sur des admissions. Il s'agit de ré-apprécier, ré-évaluer ces risques qui ont déjà été expérimentés antérieurement, depuis les contingences de ce problème spécifique là de mise en place de la nouvelle mission, et cela implique pour l'équipe tout un travail d'expérimentation d'idées et d'appréciation de leurs conséquences, travail mené à l'aune de ce qu'ils connaissent de leur pratique depuis l'expérience concrète antérieure. Ce dilemme ne peut être résolu que depuis l'expérience de l'activité : il est nécessaire de repartir de situations à comparer, mettre en rapport, en perspective dans la continuité de l'expérience ; continuité qui garantit le maillage des problèmes entre eux en les faisant avancer de façon globale dans la direction générale - la perspective - du tissu mouvant de 
la pratique. En se voyant enjoindre de mettre en place cette nouvelle mission d'insertion, les éducateurs ne peuvent donc pas «simplement» s'emparer du dispositif initialement décrit et l'appliquer tel quel, en le juxtaposant à côté de leur première mission initiale d'hébergement. Ils sont contraints de transformer le dispositif d'insertion initialement proposé pour pouvoir continuer à travailler depuis la perspective qui est la leur; notamment parce que les éducateurs et le MSP ne poursuivent pas d'emblée la même perspective ${ }^{8}$.

Le dilemme sera tranché avec la mention, dans le concept pédagogique, d'un « délai de trois mois renouvelables si nécessaire». C'est le meilleur compromis auquel sont parvenus les professionnels pour faire tenir ensemble un minimum de formalisation pour protéger la viabilité de la mission et une certaine liberté pour ajuster de façon souple le dispositif aux différents cas de figure des jeunes et garantir la pérennité du sens éducatif. Pour bien saisir les valeurs non seulement comme moyens, mais aussi comme fins dans l'activité, nous allons maintenant passer à un autre niveau de description.

\subsection{Une activité pleine de valuation pour définir pratiquement les limites du territoire}

26 Pour les éducateurs, à force d'enquêter, au fil du temps des transactions avec l'environnement se sont stabilisées : certains agencements ont produit des conséquences intéressantes en termes de visée poursuivie et ils ont alors été à nouveau activement recherchés par les professionnels, parce que leurs effets sont intéressants pour la mission. Ces régularités, qui permettent d'établir des habitudes pratiques (que nous nommions des jugements pratiques à la suite de Dewey, nous y reviendrons au point 3), dessinent un certain territoire dont les frontières sont établies à partir des risques encourus. Les risques anticipés, appréciés depuis la totalité des expériences antérieures des enquêtes, indiquent les limites du territoire pratique expérimentées et construit par les éducateurs au fil de leur expérience, les frontières par-delà lesquelles une trop grande incertitude régnerait, menaçant ainsi la viabilité de la mission dans le temps, depuis des effets mettant à mal tant la poursuite de l'activité que son sens.

À un moment de la délibération, un éducateur propose de "fixer clairement les limites institutionnelles", de "fixer les critères de tolérance qu'on peut avoir ». Il s'appuie sur une expérience inédite menée récemment auprès d'un jeune, celle-là même qui s'est finalement avérée positive après avoir rencontré de sérieuses difficultés. Il apprécie ainsi les conséquences de ce qui a été expérimenté avec ce jeune. Les limites de tolérance expriment l'idée du territoire pratique, dont il faut identifier les frontières, en référence à des situations expériencées. Cet éducateur ajoute encore que l'« on ne peut pas être complètement rigide sur ce qui est, mais qu'il faut qu'on soit clair sur ce qu'on peut tolérer ne pas tolérer ». Autrement dit il faut décider de ce qui est visé et ensuite l'ensemble des problèmes se construiront à partir de cette perspective; et à partir de là, ce qui est déterminant sont les effets des expérimentations, effets que les éducateurs ne peuvent pas connaître à l'avance. Dans cette logique de détermination a posteriori et non pas a priori de l'action, ce qui importe finalement n'est pas de savoir en amont comment on va procéder (par quels chemins on va sillonner le territoire), mais de savoir jusqu'où l'on va dans ce que l'on cherche à faire (où s'arrête le territoire, jusqu'où pousser le problème sans mettre à mal la mise en œuvre de la mission). Et les appréciations continues des professionnels dans le cours de leurs enquêtes leur permettent d'orienter l'activité en 
faisant progressivement avancer les différents problèmes dans la direction de la perspective. Elle est ce qui relie les problèmes entre eux, depuis le cumul des effets dans le temps qui fait sans cesse retentir, sur les problèmes et leur évolution, les conséquences des autres problèmes. Chaque problème est ainsi pratiquement défini dans un rapport d'interdépendance aux autres problèmes, et c'est depuis cette unité pratique, mouvante que se joue l'équilibre de la mission, en essayant de garder une prise sur les problèmes en les faisant évoluer globalement dans la même direction.

Aux limites du territoire sont attachées des valeurs comme ce à quoi tiennent les professionnels : à partir de quels moments on ne va pas plus loin dans l'intervention, non pas par décision depuis des idées absolues et idéales, mais depuis l'expérimentation de situations limites qui ne permettent plus aux éducateurs d'intervenir, à partir desquelles ils n'ont plus prise pour faire avancer les problèmes. Dans l'expérience le territoire se construit en étant sillonné de façon souple, selon des chemins qui ne peuvent pas être prédits et prédéfinis, et depuis des situations variables des jeunes à apprécier au cas par cas, mais dont les effets se cumulent depuis leur inscription pratique dans la recherche constante des mêmes effets. Et c'est depuis ce cumul des effets d'un cas à l'autre dans la polarisation de la perspective, que le territoire se construit en étant étendu et façonné progressivement dans la continuité de l'expérience. Les frontières du territoire se découvrent ainsi au fur et à mesure qu'il est construit. Comme l'exprime un éducateur, " c'est lié un peu à beaucoup de choses, qu'est-ce qui se passe quand le jeune ne va pas, qu'est-ce qu'il fait, je sais qu'il va, où est-ce que je le prends, on est obligé de penser ça en même temps qu'on pense la durée, en même temps qu'on pense les critères d'admission et puis les limites institutionnelles de tolérance ». Depuis des critères constants, mais (re)combinés différemment, singulièrement selon l'évolution des problèmes, il s'agit de favoriser au maximum la maturation des jeunes, le tout en appréciant continûment dans la longueur les effets de ce qui est expérimenté afin d'apprécier les limites par-delà lesquelles il faut éviter d'aller, sous peine de ne plus pouvoir mener une action éducative qui fasse sens en regard de la mission poursuivie.

La dynamicité du travail des valeurs dans l'immanence de l'activité n'est pas facilement ou forcément visible et appréhendable depuis le discours des professionnels. Lorsqu'elles sont évoquées de manière décontextualisée dans des discours généraux, les valeurs sont exprimées de manière absolue ne rendant pas visibles tout le travail d'enquête et le savoir-faire qu'il produit. Les valeurs ne sont pas descriptibles de façon générale sans perdre de leur consistance pratique, et d'ailleurs les éducateurs peinent à les présenter explicitement. Plus précisément, c'est leur maillage singulier dans un certain territoire pratique qui est difficilement descriptible, depuis leur inscription dans une certaine perspective. Bien entendu des choses auxquelles tiennent les professionnels affleurent directement à la surface de leurs enquêtes, comme le fait de ne pas vouloir exclure trop tôt un jeune ou au contraire de ne pas pérenniser un placement insatisfaisant ; mais ces valeurs ne sont pleinement intelligibles que prises ensemble depuis la façon dont la perspective les relie dans ce territoire pratique là. Pour exprimer ces valeurs, les formules utilisées comme par exemple "favoriser l'intégration» ou "privilégier le suivi individuel » ne nous disent rien de la façon dont ils travaillent concrètement avec ces valeurs. Celles-ci sont rapidement réduites à des formules désincarnées, peu significatives en dehors de tout engagement pratique. Cela signifie que les valeurs ne sont compréhensibles, sensibles dans leur teneur et leurs raisons d'être pratiques que depuis 
l'engagement actif dans une certaine perspective et le partage de l'expérience du territoire qui en découle.

En ce sens, les valeurs ne sont pas des "choses " nommables comme ça ou directement descriptibles, mais concrétisent des activités qui consistent tout à la fois, dans le temps même du déroulement de l'action, en des attributions situées de qualités et des directions continûment à sentir qui permettent de construire des problèmes pratiques. En enquêtant, les professionnels apprécient les relations dans lesquelles ils sont pris avec les autres ressources de l'environnement et ils leur attribuent des valeurs en regard des relations qu'ils expérimentent à cette occasion et qui constituent l'activité. Et ces appréciations des effets produits sont dans le même temps utilisées pour guider les enquêtes des professionnels en les amenant à favoriser des directions qui ont fait leur preuve jusqu'ici. Depuis ce qui a été progressivement expérimenté et apprécié comme manières de faire auxquelles se fier, c'est aussi ce à quoi tiennent les professionnels qui s'est pérennisé. Ces habitudes constituent des manières de faire stabilisées de construire les problèmes, qui sont des façons d'attribuer et de produire des valeurs. Elles donnent des directions et deviennent des guides donnant des orientations générales pour les enquêtes. Les professionnels y tiennent parce que c'est grâce à elles qu'ils ont tenu jusqu'ici. Les valeurs sont ainsi utilisées dans les enquêtes des éducateurs tant comme des moyens intervenant dans la constitution de l'activité depuis l'attribution située de qualités dans les agencements, que comme des fins guidant le travail de perspective dans les enchaînements d'agencements dans la continuité de l'action. Les valeurs sont des conduites : elles ne sont pas séparables des enquêtes et c'est là qu'opère leur production ; et pas seulement au sens instrumental où les enquêtes "utilisent» les appréciations comme moyens de connaître dans et par l'action, mais aussi au sens éthique où l'enquête produit des valeurs à l'occasion des problèmes et des objets construits.

Observer la formation des valeurs, c'est examiner des conduites en mouvement, des relations dynamiques, des séries d'action, et l'on ne peut observer directement les propriétés de cette totalité mouvante. Ce qui est observable ce sont leurs effets: les chemins pris ou non sous forme de directions, ce qui est favorisé ou non dans la continuité, les valeurs ou non attribuées aux objets comme produits des habitudes auxquelles les professionnels tiennent. Les risques à éviter sont ainsi des analyseurs pour le repérage des valeurs travaillées, formées dans le collectif, depuis le pointage des frontières du territoire comme risques à éviter. Il est possible de saisir les valeurs en creux, en les inférant depuis le territoire pratique : elles peuvent être déduites depuis le repérage des risques à éviter et des directions que les éducateurs favorisent pour s'assurer que l'action éducative ne produit pas certains risques et garde tout son sens. Du coup, décrire au terme de la recherche les valeurs observées, ce n'est pas les résumer en les exprimant ou les synthétisant à l'aide de termes généraux, mais les décrire sous forme de manière de construire et d'orienter les problèmes qui se combinent ensemble dans un certain modèle d'intervention. Dans notre démarche méthodologique, l'analyse n'est ni directe ni indirecte, mais consiste en une double modélisation empirique et théorique reconstruisant le modèle d'intervention (Mezzena, Seferdjeli, \& Stroumza, 2014 ; Mezzena \& Stroumza, à paraître), et dans laquelle nous considérons les concepts ou les idées comme des outils pour faire voir et sentir des aspects de la réalité qui resteraient inaccessibles sans ce "geste théorique du chercheur " (Friedrich, Mezzena, Stroumza, \& Seferdjeli, 2010) Il ne s'agit donc pas de plaquer une théorie sur des données empiriques, en faisant primer la théorie sur l'empirie, ou encore en cernant des problèmes empiriques 
pour ensuite aller chercher la (bonne) théorie pour expliquer ces problèmes. Dans cette perspective nous utilisons également les autoconfrontations d'une certaine manière (Stroumza \& Mezzena, 2016).

\section{Des jugements pratiques à combiner dans la perspective : le savoir-faire comme territoire pratique à équilibrer}

$\mathrm{Au}$ fil des situations, les professionnels connaissent depuis leur expérience quelles conséquences ont tendance à se déployer selon le cheminement de l'activité. Et au fil du temps, petit à petit ils savent où se situent les limites de leur intervention, quels risques anticiper. Ce qui se stabilise, ce ne sont pas des manières de faire ou des gestes en soi, significatifs individuellement, ni des actes isolés de pensée sur des actions précises, dont on pourrait extraire des compétences spécifiques. Les habitudes qui portent le savoirfaire sont des directions prises dans des séries d'activités produisant certains effets. Elles peuvent être décrites à la suite de Dewey comme des jugements9. Dans la philosophie deweyenne, les jugements stabilisés dans les enquêtes se définissent comme des équilibres de coordination entre les forces, qui sont le résultat du travail de perspective : les forces ont appris à fonctionner ensemble d'une certaine manière. Le jugement est défini comme une conduite sérielle dont le contenu se transforme au fur et à mesure des agencements, depuis une suite de rapports moyens-fins (et ne relève donc pas d'une catégorie d'activité ou d'un ensemble de situations caractérisé par une récurrence ou répétition de contenus typiques). Il aboutit à une certaine situation comme agencement qui a une durée et qui construit spécifiquement le problème en lui donnant une orientation particulière.

Notre analyse a dégagé trois jugements comme tendances actives construisant la maturation des jeunes dans une certaine direction selon les cours d'action (Mezzena, et al. , à paraître) :

- une maturation introspective, tournée vers l'intériorité du jeune, vers l'exploitation de ses ressources internes (sa sensibilité, ses émotions, sa personnalité, son expérience antérieure...);

- une maturation normative tournée vers l'extérieur et visant la conformité à des règles instituées préexistantes aux individus (règles du foyer, du marché du travail, de la société) ;

- et une maturation expérimentale tournée vers les rapports à inventer avec le monde, fondée sur les expérimentations des jeunes et leur appréciation de leurs conséquences. Aucun des jugements, pris en lui-même, n'est suffisant pour produire la perspective recherchée d'un mouvement de prise en charge du jeune par lui-même dans la durée. Selon les situations, si les éducateurs ne répondaient à ce qui se passe que depuis un seul de ces jugements, ou trop depuis le même jugement (par exemple la maturation comme introspection), l'activité n'irait pas forcément dans le sens des effets visés (par exemple les jeunes seraient amenés à être trop centrés sur eux-mêmes, ce qui risquerait à terme de favoriser plutôt de la victimisation, ou au contraire une trop grande responsabilisation avec une difficulté à exploiter des ressources extra-personnelles disponibles dans l'environnement). Comme les conduites des jeunes (et des autres forces constitutives de l'agencement) varient, les éducateurs n'ont pas la possibilité de ne s'appuyer que sur un seul jugement : pour garder le cap, l'activité les contraint à combiner les différents jugements dans la continuité de 
l'activité. C'est le seul moyen de compenser les bifurcations prises par l'activité, qui du coup n'irait plus forcément dans le sens de la perspective poursuivie pour la mission ${ }^{10}$. manière de construire les problèmes, de pouvoir passer d'un jugement à l'autre dans la continuité de l'activité que les éducateurs parviennent à maintenir un équilibre dans leur savoir-faire en dosant continûment les effets de leurs ajustements. Les éducateurs pondèrent ainsi en la transformant l'autorité éducative d'une situation à l'autre pour s'ajuster à la variabilité de ce qui se passe, et notamment celle de la conduite des jeunes, en évitant de déséquilibrer leur action éducative en la cantonnant dans des effets qui, en se cumulant, seraient ensuite trop laborieux à récupérer. Selon les situations et ce qui a précédé, orienter l'activité uniquement dans le sens d'une autorité normative ne produirait plus de mouvement de prise en charge porté par le jeune lui-même ; de même, orienter l'action éducative que dans le sens d'une introspection ne produirait que de la résistance ou serait trop lourd pour le jeune; ou encore, ne favoriser que l'expérimentation des conséquences de ses actes par le jeune ne permet pas à certains moments de mettre suffisamment rapidement une limite à des comportements impliquant une prise de risque pour soi ou autrui. Sans ces ajustements situés et continus, les effets risqueraient de ne plus favoriser le mouvement de maturation et de déporter l'activité trop en dehors de la perspective recherchée et du territoire du savoir-faire ; ce qui demanderait ensuite trop d'efforts aux éducateurs (et aux jeunes) pour transformer l'activité et ramener les conditions des situations au plus proche des effets visés. Chaque jugement trouve ainsi son efficacité dans le fait d'être combiné avec les autres, car ce sont leurs enchaînements qui sont efficaces dans la continuité. Et l'enjeu majeur pour les professionnels consiste bien à apprécier dans la continuité de l'intervention les limites des différents jugements, à quels moments il est temps d'orienter l'activité dans une autre direction. Comme l'efficacité éducative opère depuis l'appréciation d'effets distribués sur des séries d'actions, ce qui est évalué est une étendue d'effets et leur localisation dans le territoire par rapport à ses frontières. Si des situations peuvent bien entendu être appréciées isolément, l'évaluation de leur travail par les éducateurs opère cependant depuis une logique de mise en comparaison dans la continuité de l'intervention. Il ne s'agit pas de dire que tel jugement est juste ou faux (puisque seul un jugement n'est pas significatif, il ne l'est que dans les effets produits via son articulation aux autres jugements), mais d'estimer l'évolution globale des effets sur une certaine durée, depuis une appréciation approximative en fonction de la direction générale que la perspective vise.

Chacune des tendances à construire le problème de la maturation a ainsi ses avantages et ses limites selon les agencements concernés. Selon les situations, si l'activité n'est pas réorientée vers l'un ou l'autre des autres jugements de maturation, alors les limites deviennent des risques à proprement parler; risques qui, s'ils ne sont pas réduits rapidement, peuvent mettre à mal l'équilibre de la mission. Un risque est par exemple une trop grande asymétrie qui, maintenue dans la durée, peut aboutir à de la soumission de la part du jeune par peur de la sanction et verser vers une intervention coercitive, dont le mouvement entraîne le risque de s'arrêter à la sortie du foyer; ou à l'opposé, générer de la révolte se concrétisant par différentes conduites à risques comme par exemple par le repli sur soi dépressif ou des fugues. À l'autre extrême, une trop grande symétrie avec une liberté accordée au jeune fait encourir le risque d'une déresponsabilisation versant dans le laisser-aller ou l'oisiveté, voire favorisant 
l'assistance ou la victimisation. Quant au fait de les pousser à expérimenter de nouveaux rapports avec le monde, un risque se trouve plutôt dans le fait d'asservir l'éducateur au jeune, en lui laissant croire que tout est possible; avec cette idée aussi que l'enjeu consiste bien à rester dans la réalité, tout n'est pas permis sans que ne s'ensuivent des conséquences. Cette combinaison dynamique des jugements opère de façon sensible. L'enjeu majeur pour les professionnels consiste bien à sentir les limites des différents jugements, jusqu'où ils peuvent pousser et faire avancer un problème sans que la limite ne devienne un véritable risque pour l'équilibre du savoir-faire. Sentir à quels moments il est temps d'orienter l'activité dans une autre direction est ainsi au cœur du savoir-faire, même si ce travail de combinaison de jugements n'est pas effectué consciemment.

Parmi les jugements stabilisés que sont les maturations normative, introspective et expérimentale, cette dernière modalité de maturation construite comme relation à expérimenter avec le monde est la plus prisée des éducateurs, parce qu'elle permet que la relation éducative puisse être pleinement utilisée pour mettre en mouvement le jeune ; et aussi parce qu'elle s'inscrit dans une histoire institutionnelle d'un refus de l'orthopédagogie normative ${ }^{11}$ (Coenen, 2002); et enfin parce qu'il ne s'agit pas de surresponsabiliser les jeunes, dans ce lieu ceux-ci ne sont pas objets de placement pénal. Le contexte du changement de mission a permis d'observer que les éducateurs tiennent depuis leur perspective à privilégier le plus possible la maturation expérimentale, en laissant au maximum ouvert l'horizon éducatif pour favoriser les chances de faire maturer le jeune, ce qui implique concrètement, nous l'avons vu, un minimum de formalisation et le fait de ne pas restreindre l'action éducative à des outils prédéfinissant l'évolution des jeunes. Dans cette équipe-là, défendre une souplesse au niveau du dispositif éducatif se traduisant par le fait de ne pas rigidifier les règles et de ne pas (trop ou trop vite) mettre une limite temporelle à leur intervention est quelque chose à laquelle les professionnels tiennent parce que c'est la meilleure voie expériencée jusqu'ici pour favoriser au mieux la maturation des jeunes, dans l'environnement qui est le leur avec les ressources qui sont les leurs.

Notre analyse s'est enrichie depuis l'observation de l'intervention auprès d'un même jeune sur un an et demi, celui dont l'évolution s'est finalement avérée positive dans l'extrait. L'inertie des problèmes avec ce jeune, leur résistance à se transformer malgré les enquêtes des professionnels a soudain laissé la place à leur résolution : les problèmes se sont comme résolus d'eux-mêmes, ils ont tout simplement disparu. Un entretien de fin de placement avec le jeune rend visible combien la disparition des problèmes a dépendu $\mathrm{du}$ fait que ce dernier ait enfin eu confiance dans l'action éducative, qu'il ait compris que les éducateurs n'interviennent pas pour "l'embêter ", mais sont là pour le faire avancer, et qu'il embrasse alors en quelque sorte pleinement et comme naturellement la perspective des éducateurs, ce qui paradoxalement implique pour ces derniers de ne plus avoir besoin de faire des efforts pour la mettre au travail. Cette analyse nous amène aussi à constater que la nouvelle mission d'insertion a concrétisé pour ce jeune une ultime voie éducative qui est tombée à point nommé. Les éducateurs avaient tout tenté (expérimenté) avec ce jeune, sans succès (ils avaient même été jusqu'à activer l'ultime voie consistant à le placer en observation quelques jours en milieu carcéral pour adolescents).

Les valeurs sont le produit des jugements. Les jugements ne sont donc pas détachables des valeurs qu'ils construisent et qui sont intrinsèques au travail d'enquête. Les valeurs se forment en évoluant depuis l'expérimentation de situations nouvelles et leur formation, qui implique malgré tout une stabilisation même si elle est toujours provisoire, opère 
collectivement depuis l'expérimentation d'effets communs dans les enquêtes. Ce qui est communément partagé dans le collectif, ce ne sont pas des idées, des conceptions ou encore des représentations opératoires communes, considérées comme justes et vraies et sur lesquelles se mettre d'accord (d'ailleurs on ne constate pas un consensus à ce niveau dans l'équipe, et en outre on observe une pluralité chez les professionnels eux-mêmes). L'accord dans l'action opère depuis les conséquences de ce qui est expérimenté dans la construction des problèmes (Mezzena, et al., 2016; Mezzena, et al., à paraître): les éducateurs se retrouvent dans le partage des effets de leurs expérimentations, en faisant tous communément l'expérience d'effets identiques, même s'ils s'y prennent ou voient le problème différemment. Ces conséquences partagées sont des directions que les professionnels cherchent à favoriser depuis leur perspective, ou de manière concomitante comme des risques que les professionnels cherchent constamment à éviter. Ce qui fait la cohérence de l'action éducative n'est pas le fait de défendre tel ou tel contenu (d'idées, de principes, de prescriptions...), mais d'agencer des valeurs comme choses auxquelles on tient, en réorientant l'activité selon la particularité des agencements concernés, de sorte à parvenir à favoriser certains effets dans le temps et à faire perdurer une certaine direction à l'activité pour garantir la poursuite de la mission.

Équilibrer constamment la perspective, parvenir à favoriser ce à quoi on tient en privilégiant certaines directions depuis la combinaison des jugements, relève d'un travail de tous les instants pour ré-ajuster l'orientation de ce qui se passe. Nous avons affaire à une unité faite d'une certaine cohérence que l'on peut qualifier d'opérationnelle, parce qu'elle relève de critères pratiques, expérientiels. La cohérence du savoir-faire a à voir avec l'orientation générale prise par l'activité et la cohérence relève alors non pas de contenus dans des actions prises isolément, mais plutôt des directions prises permettant de ne pas sortir (trop longtemps) du territoire et de ses limites. Ainsi, dans le modèle que le savoir-faire fabrique, la pluralité n'est pas un problème, au sens où il ne devrait y avoir qu'une seule (bonne) manière de construire les problèmes pratiques. Au contraire, c'est bien parce qu'il y a différentes manières possibles de construire les problèmes pratiques que l'activité peut absorber la variabilité et faire avec l'incertitude sans mettre à mal la mission et son équilibre. La cohérence du coup n'est pas une affaire de manières de faire justes ou fausses, ou cohérentes ou pas assez cohérentes en soi en regard de critères absolus, mais de continuité dans la direction favorisée depuis le cumul des effets. Ce qui est incohérent serait de laisser une direction s'installer dans la durée pour la construction des problèmes, alors qu'elle ne produit pas les effets recherchés et fait alors courir le risque de déséquilibrer l'agencement des jugements dont la combinaison a fait ses preuves jusqu'ici.

40 Selon la façon dont se combinent les jugements, l'activité déporte le travail éducatif vers d'autres régions du territoire de l'activité. D'où l'idée essentielle que dans les enquêtes nous n'avons pas affaire à des catégories d'activités ou des familles de situations, mais à des étendues de rapports sur un territoire comme totalité à équilibrer. Dans l'activité en train de se dérouler, les trois manières de construire la maturation s'enchainent sans cesse, et c'est dans cette sérialité ou continuité de l'activité qu'une certaine tendance, à un moment donné, devient prédominante et prend en quelque sorte le dessus en donnant provisoirement une direction à la définition de la maturation. C'est donc en examinant ce que devient l'activité dans la continuité que se dessine pour un temps une certaine orientation. D'où l'impossibilité de découper l'activité en situations qui se juxtaposent et que l'on pourrait typifier (telle situation relevant de tel jugement et donc de telle 
dynamique de maturation). De plus, les jugements ne sont pas attribués en propre et de manière personnelle aux professionnels. Chaque professionnel travaille à construire la maturation depuis ces trois jugements. Les jugements ne renvoient pas non plus à des idées finies et autosuffisantes, que l'on pourrait mettre en correspondance avec des problèmes typiques. Les jugements peuvent être compris comme des suites ou des chemins d'agencements dont les significations s'enrichissent au fil des nouveaux rapports et de leurs effets, tout en étant globalement orientées dans des directions convergentes.

41 Ainsi la signification globale de la perspective de la maturation depuis la combinaison dynamique des trois jugements ne peut être saisie de manière intelligible que dans ce contexte d'intervention-là. Ensemble, en s'agençant, les jugements forment un certain modèle de savoir-faire (de Jonckheere, 2010), qui n'est pas réductible à un modèle de nature théorique, mais est au contraire un produit de l'activité. Si ce modèle d'intervention que fabriquent les professionnels est progressivement construit dans l'activité depuis le travail de mise en perspective, à son tour, au fil du temps, il devient un guide pour les enquêtes futures, depuis les jugements stabilisés comme habitudes de partenariat avec l'environnement.

La reconstruction du modèle du savoir-faire nous amène aux constats suivants concernant les valeurs dans l'activité des éducateurs du foyer étudié, valeurs éducatives qui vont guider leurs enquêtes pour mettre en place la nouvelle mission. C'est le cumul de plusieurs situations d'impuissance comme celle-ci qui a rendu nécessaire et légitime l'ajout de cette deuxième mission, et cette opportunité nouvelle a offert une issue éducative potentielle supplémentaire qui dans ce cas a été une réussite. Nous observons cependant qu'il y a une condition pratique incontournable pour cette réussite : c'est que ce dispositif d'insertion, a priori déjà tout pensé et conçu dans le projet initial, fasse l'objet d'une reconstruction complète depuis la perspective poursuivie dans ce lieu-là. Dans cette équipe, un enjeu fort pour tenir dans les activités éducatives, aussi bien pour les jeunes que pour le MSP, consiste à ce que le dispositif ne soit pas habité depuis une autre perspective (notamment celle de l'orthopédagogie normative). En observant que les éducateurs continuent à privilégier la maturation expérimentale, nous constatons aussi que le changement de mission n'a ni déplacé la perspective, ni fondamentalement transformé les habitudes de partenariat avec l'environnement (ce qui ne signifie pas qu'il n'y a pas de changement du tout). Ce résultat ouvre une nouvelle piste encore à creuser sur la question de l'innovation dans les pratiques éducatives, qui peut être présentée comme opérant de manière diffuse et pas forcément consciente, et qui nécessite que les outils, dispositifs ou méthodes importés depuis le champ éducatif ou souvent en travail social depuis d'autres champs professionnels (notamment thérapeutiques) soient complètement revisités, ré-agencés pour satisfaire l'orientation de la perspective propre à cette équipe-là, à ce moment-là dans ce champ-là.

\section{La connaissance dans l'activité : une connaissance de nature pratique}

43 Notre approche de l'enquête comme valuation nous apprend que dans l'expérience, valeurs et connaissances s'intègrent sous forme d'idées tantôt utilisées pour expérimenter des rapports dans les agencements constitutifs de l'activité, tantôt utilisées comme des guides orientant le travail de perspective. Depuis la définition immanente des valeurs comme conduites, les valeurs ne sont plus séparées de l'expertise professionnelle, 
mais au contraire y participent pleinement en nourrissant et en guidant le travail de perspective.

Mais du coup, c'est la question du rapport à la connaissance qui se (re)pose, car en effet, nous n'avons plus alors affaire à une opposition entre valeurs comme croyance subjective d'un côté et connaissance comme vérité objective de l'autre. Dans l'activité, que l'on parle de valeur ou de connaissance, nous observons qu'elles existent en s'actualisant autant comme moyens que comme fins. Ce double statut logique et la non-distinction empirique qui en découle nous amènent à proposer plutôt la notion d'idée pour évoquer dans le processus d'enquête l'usage d'idées tant dans les agencements sous forme d'ajustements, que dans le travail de perspective. Les valeurs définies comme des conduites et observées comme telles dans le travail d'enquête nous amènent donc à constater que la séparation ou le dualisme entre connaissance et valeur n'est pas pertinent d'un point de vue épistémologique, ce qui nous conforte dans le fait de ne pas vouloir séparer les valeurs de l'expertise professionnelle.

Ce résultat nous amène à définir le savoir-faire comme étant diffus et comme relevant d'une connaissance de tous les instants (ou ordinaire selon la formule d'Ogien (2014): c'est l'appréciation d'effets diffus, distribués sur des séries de situations, sur des chemins de mises en rapport et le tout dans la continuité de l'expérience, qui permettent aux éducateurs de sentir quelles directions, quels jugements de maturation ils ont meilleur temps de favoriser pour maintenir l'orientation dans le sens des effets recherchés (Mezzena, et al., 2016 ; Mezzena, et al., à paraître ; Mezzena, à paraître).

Notre modélisation située et continuiste de l'activité remet en question le paradigme réflexif de la compétence professionnelle, largement naturalisé dans les pratiques de formation en alternance des hautes écoles professionnelles des métiers de l'humain de Suisse romande (Mezzena, à paraître). Issu du modèle du praticien réflexif de Schön $(1983 / 1994 ; 1996$ a et b), ce paradigme définit la professionnalité comme mise à distance, analyse et formalisation ou conscientisation de l'action. Il partage avec la vision managériale du travail les présupposés d'un professionnel positivé, pouvant maîtriser son action et ses effets depuis un travail réflexif d'analyse de l'action. Son ressort consiste en une réflexion dans et sur l'action permettant aux professionnels de mettre en correspondance des situations-problèmes d'un côté et des idées de l'autre, via le truchement, à partir d'une analyse de ce qui se passe, de connaissances à retrouver et à appliquer sur le problème. La connaissance y est ainsi définie, depuis un cognitivisme classique ou sa version plus modérée du représentationnalisme minimaliste (Steiner, 2010), comme des contenus stockés dans le mental sous forme de représentations opératoires. Ce paradigme réflexif peut également être rattaché à une certaine épistémologie également très diffusée dans les recherches en sciences de l'éducation, présupposant un sujet posé face à des objets dans l'environnement, dans un rapport d'adaptation (et non pas de transformation) à ce dernier (Durand, Poizat, \& Goudeaux, 2015).

Depuis le travail d'enquête, nous constatons que la connaissance est une affaire de sérialité et de direction dans des activités dans la continuité de l'expérience. Ce qui fonde la connaissance, ce qui permet de fonder des certitudes sur la pratique, est ainsi déplacé de contenus "justes » à trouver ou retrouver «dans la tête " par le truchement de la réflexion (et donc additionnel à la pratique), à des mises en rapport situées et dynamiques dans l'activité même entre faits idées, rapports à expérimenter comme moyens pour construire des problèmes sur lesquels pouvoir opérer. Dans le travail de perspective 
permettant d'orienter l'évolution des problèmes dans le sens de la mission, nous avons affaire à un travail de "sentir " plutôt qu'à un " réfléchir » ou de la réflexion. Il s'agit en effet de sentir continûment la direction que prennent les problèmes en rapport avec la totalité de l'expérience antérieure de la construction du territoire (Mezzena, et al., 2016). Ce résultat permet de trouver du sens aux descriptions des professionnels, qui parlent constamment de sentir les situations. Ce sentir comme savoir-faire aux propriétés sensibles et non réfléchies opère depuis les habitudes de partenariat avec l'environnement. Ils sentent directement l'agencement ou les rapports aux autres forces en termes de direction prise et peuvent s'ajuster sans réfléchir, au sens ici d'un rapport conscient à soi-même ou d'un effort d'analyse consciente et de mise à distance de l'action.

Nos observations nous ont ainsi amenés à explorer une alternative à la définition représentationnaliste de la connaissance (Mezzena, à paraître; Mezzena, et al., 2016). Depuis les travaux de Steiner dans le champ de la philosophie de la cognition (2010; à paraître), ceux d'Ogien dans la sociologie pragmatiste de l'action $(2015 ; 2014)$ et ceux de de Jonckheere également d'obédience pragmatiste dans le champ du travail social (2010), les connaissances peuvent être définies de manière non mentaliste, non pas comme des contenus stockés dans le mental, mais comme des activités continues de mis en rapport. Avec ces auteurs, nous pouvons ainsi concevoir la connaissance comme une activité de mises en rapport dynamique, comme de la relationnalité mettant en rapport des idées avec des faits pour construire des problèmes.

Cette définition de la connaissance comme activité de construction de relations a une conséquence importante : il ne s'agit plus de dire que les connaissances fondent l'activité et donc la pratique en exprimant des vérités à elles seules, mais bien plutôt qu'elles accompagnent la construction des problèmes (de Jonckheere, 2010 ; Mezzena, à paraître). D'une situation à l'autre, les agencements sont remis à l'épreuve et leur signification possible est redéveloppée depuis les expérimentations incessantes et l'appréciation de leurs effets. Des connaissances qui ont été des idées comme guides sous forme de relations moyens-fins se trouvent ainsi transformées depuis leur usage comme moyens. Dans l'usage situé et continu de la connaissance comme chemins de rapports sillonnant un territoire pratique, il y a donc continûment du développement et de l'approfondissement, depuis les transformations continues que l'action fait subir à la connaissance comme agencement d'idées et de faits. En tant que résultats d'enquête, les connaissances existent sous forme d'idées généralisées de fins et de valeurs qui orientent les enquêtes dans la poursuite de la perspective. Il s'agit de connaissances auxquelles les professionnels tiennent et qui ont une valeur, parce qu'elles ont fait leur preuve et qu'ils ont pu s'y fier jusqu'ici. Il y a là un apport central de la pratique comme lieu d'expérimentation et d'enrichissement des idées, en partenariat avec l'environnement.

Nos résultats au sujet de la connaissance nous ont aussi amenés à nous tourner vers James, autre philosophe pragmatiste, pour développer davantage encore la notion d'expérience que ne le fait Dewey, et cela concrétise une nouvelle piste encore à travailler. Ce qui fonde la connaissance est la façon dont des effets intéressants sont produits pour la perspective, ou comme dirait James (2007) selon la différence que cela fait pour l'expérience. Dans les enquêtes pratiques, nous n'avons donc pas uniquement affaire à une logique d'accumulation de connaissance au sens quantitatif du terme, mais à une logique conséquentialiste et transformatrice comme connaissance "déambulatoire " qui opère de proche en proche comme construction de relations dans des contextes 
d'activités et qui nous permet de «faire connaissance» (James, 2007; Stroumza \& Mezzena, 2016).

Dans l'approche pragmatiste que nous avons privilégiée, la connaissance procède donc de l'expérience et non l'inverse. La professionnalité n'est pas d'abord conçue comme un cumul de contenus stockés dans le mental sous forme de représentations opératoires et formant un référentiel commun dans lequel puiser réflexivement les bonnes réponses pratiques après analyse des situations, en conformité à des prescriptions, normes, théories ou réflexions extérieures à l'action. La professionnalité se déplace du professionnel à l'activité, ou encore de contenus à appliquer présentés comme des vérités absolues à des conséquences comme effets des enquêtes. D'où au final une professionnalité de nature publique, située et dynamique (Mezzena, et al., 2016) relevant d'une connaissance ordinaire, non pas eu sens d'une non-expertise, mais au sens d'une connaissance de tous les instants dans la continuité de l'expérience ; une professionnalité non pas cachée dans l'action sous forme de savoirs propositionnels, théoriques à retrouver, mais diffuse et exprimée sous forme de mises en rapport dynamique et continus, et se déployant dans un territoire pratique spécifique.

\section{BIBLIOGRAPHIE}

Aballéa, F. (Ed.). (2012). Institutionnalisation, désinstitutionnalisation de l'intervention sociale. Toulouse : Octarès Éditions.

Bidet, A., Quéré, L., \& Truc, G. (2011). Ce à quoi nous tenons. Dewey et la formation des valeurs. In J. Dewey, La formation des valeurs (pp. 5-64). Paris : La Découverte.

Brichaux, J. (2001). L'éducateur spécialisé en question(s). La professionnalisation de l'activité socio-éducative. Paris : Erès.

Coenen, R. (2002). Éduquer sans punir. Vers une approche sociothérapeutique de l'adolescence et de la délinquance. Thérapie Familiale, 23(4), 325-348.

De Zotti, P., Dumont, J.-F., \& Marty, P. (2007). Regards croisés sur la professionnalisation. Enjeux et perspectives. In C. Mias \& M. Lac (Eds.). Travail social et formation : voies pour la professionnalisation, Revue Internationale des Sciences de l'Éducation, 17, 51-64.

Dewey, J. (1938/1993). Logique. La théorie de l'enquête. Paris : PUF.

Dewey, J. (2011). La formation des valeurs. Paris : La Découverte.

Durand, M., Poizat, G., \& Goudeaux, A. (2015). Individuation, pensée de la formation et technologie éducative. Une lecture de Simondon selon une perspective enactive et développementale. In J. Baillé (Ed.), Du mot au concept : l'individu (pp. 119-145). Grenoble : PUG.

Garreta, G. (2007). L'éthique située de Dewey. Émotions, états d'esprit et sens des jugements moraux. L'Art du Comprendre, 16, 27-48.

James, W. (2007). Le pragmatisme. Paris : Flammarion.

Joas, H. (1999). La créativité de l'agir. Paris : Éditions du Cerf. 
Jonckheere de, C. (2007). Éthique et activité dans le travail social. In J. Libois \& K. Stroumza (Eds.) Analyse de l'activité en travail social (pp. 105-128). Genève : Éditions ies.

Jonckheere de, C. (2010). 83 mots pour penser l'intervention en travail social. Genève : Éditions ies.

Lansiaux, V. (2008). Le métier d'aide médico psychologique. Comment former des professionnels dans un contexte concurrentiel ? In Nouvelles configurations des métiers du social. Travail emploi formation, cahiers de sociologie et d'économie régionale, 8, 50-59.

Mezzena, S. (2012). Engagement des travailleurs sociaux depuis le partenariat avec l'environnement : agencements et formation des valeurs. Revue Forum, 136, 37-46.

Mezzena, S. (à paraître). Une connaissance pratique logée dans l'activité même. Discussion critique du praticien réflexif de Schön depuis la théorie de l'enquête de Dewey (titre provisoire), Genève : Éditions ies.

Mezzena, S., \& Stroumza, K. (à paraître). Imprévisibilité de l'action et intelligence pratique : quelques éléments de méthodologie pour l'analyse de l'activité. Revue AFFUTS.

Mezzena, S., Stroumza, K., \& Kramer, N. (2016). L'insertion des adolescents en difficulté : situations douteuses, art d'hésiter et connaissance pratique dans l'activité des éducateurs. Sciences et actions sociales, 4. http://www.sas-revue.org/index.php/17-varia/70-l-insertion-desadolescents-en-difficulte-situations-douteuses-art-d-hesiter-et-connaissance-pratique-dans-lactivite-des-educateurs.

Mezzena, S., Stroumza, K., Kramer, N., \& Baumgartner, P. (à paraître). Connaissance, valeur et professionnalité dans la pratique comme territoire à équilibrer. Enquêtes et perspective dans l'activité des éducateurs (titre provisoire).

Mezzena, S., Stroumza, K., \& Seferdjeli, L. (2014). Des recherches qui prennent pour objet les savoir-faire. In D. Paturel (Ed.), Recherche en travail social : les approches participatives (pp. 220-235). Nîmes : Champ Social.

Mezzena, S., Stroumza, K., Seferdjeli, L. \& Baumgartner, P. (2013). De la réflexivité du sujet aux enquêtes pratiques dans l'activité d'éducateurs. Activités, 10(2), http://www.activites.org/v10n2/ v10n2.pdf.

Ogien, A. (2010). La valeur sociale du chiffre. La quantification de l'action publique entre performance et démocratie. Revue française de socio-économie, 5, 19-40.

Ogien, A. (2013). Qu'est-ce qui fait valeur dans notre société ? Archimède, 8-10.

Ogien, A. (2014). Revenir à l'ordinaire. L'exercice de la connaissance en situation d'intervention. Raison publique, 18, 77-91.

Ogien, A. (2015). Quand agir, c'est penser. Certitude, conviction et connaissance directe. Intellectica, 63, 15-35.

Quéré, L. (2006). L'environnement comme partenaire. In J.-M. Barbier \& M. Durand (Eds.) Sujets, activités, environnements. Approches transverses (pp. 7-29). Paris : PUF.

Ravon, B. (2008). Comment traverser les épreuves du travail social ? Construction de la professionnalité et dispositifs d'analyse de la pratique. Rhizome - Bulletin national santé mentale et précarité, 33, 48-51.

Schön, D. A. (1984/1993). Le praticien réflexif. À la recherche du savoir caché dans l'agir professionnel. Montréal : Éditions Logiques. 
Schön, D. A. (1996a). À la recherche d'une nouvelle épistémologie de la pratique et de ce qu'elle implique pour l'éducation des adultes. In J.-M. Barbier (Ed.), Savoirs théoriques et savoirs d'action (pp. 201-222). Paris : PUF.

Schön, D. A. (1996b). Le tournant réflexif. Montréal : Éditions Logiques.

Sorel, M. (2005). Alors, professionnaliser les formations ? In M. Sorel \& R. Wittorski (Eds.), La professionnalisation en actes et en questions (pp. 159-170). Paris : L'Harmattan.

Steiner, P. (2010). Quelques arguments pour un minimalisme représentationnel. Forthcoming in INCognito. Cahiers Romans de Sciences Cognitives, 4(3).

Steiner, P. (à paraître). Quelques arguments pour un minimalisme représentationnel. Cahiers Romans de Sciences Cognitives, 4(3).

Stroumza, K., \& Mezzena, S. (2016). «Faire connaissance », « se faire penser » : un dispositif d'autoconfrontation dans une démarche de visibilisation des savoir-faire des modérateurs dans les bus nocturnes. Raisons éducatives, 20, FPSE, Université de Genève.

Stroumza, K., Mezzena, S., Friedrich, J., \& Seferdjeli, L. (2014). L'ajustement dans tous ses états : règles, émotions, distance et engagement dans les activités éducatives d'un centre de jour genevois. Genève : Éditions ies.

\section{NOTES}

1. Ce projet de recherche intitulé, "Formation des valeurs dans l'activité et construction de la professionnalité des éducateurs", a été financé par le Fonds national suisse de la recherche scientifique (FNS) de 2013 à 2015.

2. Nous retrouvons ici la conception déterministe classique des valeurs en sociologie comme "principe (moral ou politique) figé dans une "culture" qui le transmettrait immanquablement à un individu qui l'intériorise et règle mécaniquement ses conduites ou ses attitudes sur ce qu'il prescrit »; valeur qui tiendrait au fait d'être "une représentation certifiée et concise d'une réalité objective » (Ogien, 2010, p. 20).

3. Nous nous inscrivons ainsi bien dans la mouvance des travaux présentés dans le dossier «Pragmatisme et activités : des interactions aux transactions» du numéro 10-1 de la revue Activités.org (2013).

4. Dans notre modélisation de l'activité, nous opérationnalisons ce postulat du partenariat avec l'environnement avec les notions de forces, d'ajustement et d'agencement (Mezzena, 2012) : les «entités» présentes dans l'environnement, humaines ou non, répondent aux actions des professionnels en s'y ajustant d'une certaine manière, tout comme ceux-ci y répondent également (Stroumza, Mezzena, Friedrich, \& Seferdjeli, 2014). L'activité est issue de ces ajustements mutuels qui ensemble forment des agencements de forces. A la suite de Quéré (2006), nous définissons les agencements comme des équilibres de coordination entre forces de nature différente qui doivent travailler ensemble; et en se répondant, des effets sont produits qui amènent l'activité à se réorganiser en cours de route, de manière interne au fil de l'enchaînement de leurs rapports mutuels. Dans cette définition située de l'activité, il s'agit pour les professionnels de ne pas se laisser complètement déterminer dans et par ce jeu de forces, d'où tout un travail d'enquête comme enchevêtrement d'expérimentation, d'appréciation et d'anticipation qui opère dans la continuité de l'expérience afin de tenter d'influencer l'orientation de l'activité dans le sens des effets recherchés pour la mission. Les enquêtes pratiques permettent aux professionnels de construire et faire évoluer leurs problèmes depuis une régulation en continu et a posteriori des effets produits par les expérimentations (Mezzena, 
Stroumza, Seferdjeli, \& Baumgartner, 2013 ; Mezzena, Stroumza, Kramer, \& Baumgartner, à paraître).

5. Dewey (1938/1993) définit la situation comme un tout qualitatif expériencé, senti et diffus, faisant prendre une certaine direction à l'activité.

6. Cette pérennisation dans l'orientation de la construction des problèmes pratiques est rendue possible pas seulement parce que les professionnels le désirent en regard des besoins à satisfaire, mais surtout parce que l'environnement comme partenaire le permet depuis les ressources qu'il met à disposition et la plasticité dont il fait preuve dans les transactions.

7. Dans les enquêtes, l'anticipation de risques permet d'éviter que des événements ne mettent (trop) à mal l'activité et son devenir. D'où une conception nécessaire et positive du risque comme «opération épistémique » intrinsèque à «l'exercice de la connaissance en situation d'intervention » (Ogien, 2014) et constitutive du savoir-faire.

8. Sans pouvoir développer ici la comparaison entre ces deux perspectives, notons par exemple que si les éducateurs tiennent au fait de ne pas trop formaliser le dispositif des ateliers afin de garder un maximum de marge de manœuvre, le MSP quant à lui rejoint cette équipe en concevant la mise en place de l'atelier de production avec des outils formalisant l'évolution des jeunes depuis des grilles préétablies décrivant la conduite des jeunes à l'aide de catégories et d'indicateurs prédéfinis.

9. Dans sa théorie de l'enquête, Dewey définit les jugements non pas de façon mentaliste et intellectualiste comme des idées finies et autosuffisantes, mais comme de nouvelles manières de construire des situations au terme d'enquêtes (Mezzena, à paraître); situations qui, il faut le rappeler, ne sont pas statiques mais comprennent une durée. Les situations sont synonymes d'agencements d'idées et de faits qui, depuis l'obtention d'un certain équilibre de coordination entre les forces constitutives de l'activité, orientent celle-ci dans une certaine direction. Nous retrouvons ici la définition des idées chez James (2007), pour qui il s'agit de guides.

10. Ce qui peut arriver par moment est que les effets fassent sortir l'activité du territoire et de sa perspective, mais cela ne doit pas durer au risque de mettre en péril le devenir de la mission (ce qui ne doit pas être entendu comme le fait de ne plus parvenir à combiner les différents jugements, car dans les faits ces trois jugements sont toujours activement présents d'une manière ou d'une autre dans le guidage de l'activité).

11. Par « orthopédagogie normative », Coenen entend «toute pédagogie du redressement; tout système pédagogique reposant principalement sur le respect de règles de «bon comportement » édictées par un règlement d'ordre intérieur; et dont les outils pédagogiques visent préférentiellement la soumission à ce règlement par la menace, la punition, le chantage au renvoi, la délation à l'autorité »(Coenen, 2002, p. 327).

\section{RÉSUMÉS}

Dans une recherche consacrée à la construction de la professionnalité depuis l'expérience de l'activité, nous interrogeons la formation des valeurs dans les enquêtes pratiques d'éducateurs spécialisés dans un foyer pour adolescents en rupture familiale et sociale. Dans un contexte de dédoublement de mission depuis l'injonction à créer des ateliers d'insertion, nous analysons la façon dont les professionnels sauvegardent pratiquement certaines valeurs auxquelles ils tiennent, en se confrontant à des dilemmes posés par cette évolution de leur mission. C'est le rôle et la place des valeurs comme guides depuis la stabilisation de manières de construire les 
problèmes pratiques qui sont examinés. Une analyse de délibérations en colloque est l'occasion d'observer combien les appréciations des professionnels, qui consistent à attribuer en situation des valeurs aux relations avec les autres objets de l'environnement, sont incessantes et déterminantes pour la poursuite de leur perspective dans leurs enquêtes pratiques. Ce constat ouvre une voie pour problématiser les valeurs de façon non absolutiste sans les opposer à l'expertise ou à la technicité professionnelle. Dans notre approche située et continuiste du savoir-faire, valeurs et connaissances tiennent ensemble dans le processus d'enquête construisant la professionnalité. Nous sommes ainsi amenés à discuter la définition de la connaissance, en la considérant comme une activité de mise en rapport située et continue, de nature pratique et non pas intellectualiste sous forme de contenus stockés dans le mental. Pour finir, nous tirons des conclusions pour la définition de la professionnalité.

In our research on the construction of professionality derived from professional activity, we examine the development of values based on practical inquiries of special educators working in a home for adolescents whose family and social situations have broken down. In the context of a dual mission, based on creating integration workshops, we analyze in practical terms how the professionals retain certain of their values by confronting the dilemmas they face during the fulfillment of their mission. We examine the role and place of values as situated conducts that we conceive to be guides for stabilizing ways of constructing practical problems. An analysis of discussions at symposia provides the opportunity to observe the extent to which professionals' assessments, which consist in assigning value to the relationships with other objects in the work place, are continuous and critical to the activity. They allow a continuous search for effects as a perspective from which to orientate their practical inquiries. This finding provides a means to problematize values in a manner which is not absolutist and which does not bring them into conflict with professional expertise or technicality. In our situated approach, which focuses on a developing set of skills, values and knowledge are combined in the inquiry process that builds professionality. This leads us to consider, in particular, the classic definition of representational knowledge. Finally, we draw conclusions regarding the definition of professionality.

\section{INDEX}

Mots-clés : valeurs, connaissance, colloque, dilemme, professionnalité des éducateurs

Keywords : values, knowledge, colloquium, dilemma, professionality of special educators

\section{AUTEURS}

\section{SYLVIE MEZZENA}

Haute école de travail social - HETS//HES-GE (https://www.hesge.ch/hets/), 28 rue PrévostMartin, Case postale 80, CH-1211 Genève 4, sylvie.mezzena@hesge.ch

\section{KIM STROUMZA}

Haute école de travail social, 28 rue Prévost-Martin, Case postale 80, CH-1211 Genève 4, kim.stroumza@hesge.ch

\section{NICOLAS KRAMER}

Haute école de travail social, 28 rue Prévost-Martin, Case postale 80, CH-1211 Genève 4, nicolas.kramer@hesge.ch 